\title{
La traducción del lenguaje de los ochenta en Stranger Things
}

\section{Translating the language of the eighties in Stranger Things}

\author{
IRENE RUIZ SAN MIGUEL \\ LUCILA MARÍA PÉREZ FERNÁNDEZ \\ irene.ruizsm@gmail.com \\ Lucila.perez@uneatlantico.es \\ Universitat Autònoma de Barcelona \\ Universidad Europea del Atlántico
}

Fecha de recepción: 29 de octubre de 2018

Fecha de aceptación: 5 de febrero de 2019

Resumen: El presente artículo trata sobre la variación diacrónica y su traducción. En él nos centramos concretamente en la traducción al español de un fenómeno audiovisual a nivel internacional, la serie estadounidense Stranger Things (dirigida por Matt y Ross Duffer, 2016-2019). Analizamos los términos propios del lenguaje de los años ochenta en la cultura estadounidense en la que se ubica la serie y su traducción en la versión española, tanto de la primera como de la segunda temporada. En el análisis podremos ver por medio de corpus, diccionarios y otra variedad de recursos el uso, las épocas y el registro de los términos, tanto en español como en inglés. Asimismo observaremos las dificultades que supone la traducción de la variedad diacrónica, especialmente con un producto audiovisual como este, dado que trae consigo limitaciones ligadas al código de colocación de sonido, tanto de isocronía como de sincronización.

Palabras clave: variación diacrónica, traducción audiovisual, años ochenta, cultura estadounidense, corpus

Abstract: The subject of the present study is diachronic variation and its translation. Specifically we focus on the Spanish translation of the international audiovisual phenomenon that the American TV series Stranger Things represents. We analyze the terminology characteristic of the language of the eighties in the American culture where the series is placed, as well as the translation of the Spanish version, both in the first and second season. Through the analysis we will be able to see - thanks to corpora, dictionaries and other kind of resources - the use, period and register of such terminology, both in English and Spanish. Moreover, we will observe the difficulties entailed when translating diachronic variation, especially with 
an audiovisual product like this one, since it involves restrictions related to sound code, both isochrony and phonetic synchronization.

Keywords: diachronic variation, audiovisual translation, eighties, American culture, corpora

\section{INTRODUCCIÓN}

Bien es sabido que el proceso traductor exige del profesional no solo el conocimiento de dos lenguas, sino también de las culturas y sociedades asociadas a estas. Existen diversos factores a los que el traductor, sin importar el tipo de encargo, debe prestar atención siempre. Estos incluyen las variedades dentro una misma lengua, los aspectos sociológicos y culturales de ambas lenguas, el contexto en el que se desarrolla, los aspectos ideológicos o políticos del texto original, así como la finalidad e intención de la traducción, entre muchos otros. A todos estos factores, habituales como decimos en la mayoría de encargos de traducción, se puede sumar un nuevo aspecto que encontramos principalmente en el ámbito de la traducción literaria y audiovisual: la traducción de la variedad diacrónica, es decir, la traducción del lenguaje (y la cultura que va unida a este) de otras épocas.

En el presente artículo analizaremos el caso de la traducción de la variedad diacrónica en el doblaje en español de la serie Stranger Things. Este estudio se enmarca dentro del ámbito de la traducción audiovisual (en adelante TAV), una disciplina madura cuya investigación ha avanzado notablemente en el siglo actual (Chaume, 2018). En él nos centraremos en el estudio de la traducción del lenguaje de los años ochenta: ¿Cómo traducimos la variación diacrónica? ¿De qué recursos dispone un traductor para conseguir traducir el lenguaje de otra época en la que puede que ni siquiera haya vivido?¿Cuáles son las limitaciones en este tipo de traducciones?

\section{LA TRADUCCIÓN AUDIOVISUAL}

De acuerdo con Mayoral (2001, p. 20) el estudio de la traducción audiovisual tiene su origen en el estudio de la traducción cinematográfica. Agost (1999, p. 15) define esta modalidad de traducción como una «traducción especializada que se ocupa de los textos destinados al sector del cine, la televisión, el vídeo y los productos multimedia». De acuerdo con esta autora se trata de un tipo de traducción con unas características específicas, pues requerirá profesionales con conocimientos no solo del 
campo temático sobre el que verse el producto, sino también sobre las peculiaridades, limitaciones y técnicas características que condicionan la traducción.

Respecto a las características de la traducción audiovisual, Agost (1999, p.15) explica:

Este tipo de traducción tiene unas características propias, ya que exige del profesional unos conocimientos especiales, no tan solo por el campo temático (el contenido) -que puede ser múltiple-, sino especialmente por las limitaciones y las técnicas particulares que se utilizan y que condicionan la traducción.

Las limitaciones a las que hace referencia la autora son varias, y estas están ligadas a los canales y códigos involucrados en este tipo de traducción. Los productos audiovisuales son «aquellos productos de comunicación que se sirven de señales auditivas (diálogo, narración, música, efectos) y de señales visuales (imágenes, texto narrativo, subtítulos) para transmitir un mensaje» (Mayoral, 1998, p.1) y se transmiten por tanto por dos canales: el acústico y el visual, de forma simultánea.

Canal acústico (las vibraciones acústicas a través de las cuales recibimos las palabras, la información paralingüística, la banda sonora y los efectos especiales) y el canal visual (las ondas luminosas a través de las que recibimos imágenes, pero también carteles o rótulos contextos escritos, etc.) (Chaume, 2004, p. 30).

Esto hace que, en consecuencia, la traducción audiovisual sea una traducción subordinada, ya que la función del traductor es adaptar el código lingüístico, el texto, respecto a un código visual que permanece invariable (Hurtado, 2001).

Sin embargo, pese a todas las restricciones que aparentemente supone la traducción audiovisual y a las que se refieren multitud de expertos en la materia, resulta interesante el punto de vista que ofrece Martínez Sierra (2001). Este autor defiende que, aunque el contexto visual puede suponer una restricción, también puede ayudar, al espectador y al traductor, a entender lo que está ocurriendo en la pantalla.

Si bien nosotras nos estamos refiriendo a este proceso de traducción de productos audiovisuales como traducción audiovisual, es importante señalar que existen otros autores, como indica Díaz Cintas (2001, pp. 2324), que consideran que sería más correcto utilizar el término adaptación debido a las múltiples limitaciones que esta tarea supone en el medio audiovisual. Sin embargo, tanto Díaz Cintas, como otros teóricos como 
Mayoral, contemplan una idea de la traducción más amplia y flexible, y por ello consideran el término traducción audiovisual correcto.

\subsection{La traducción audiovisual y el doblaje}

Desde sus inicios a principios del siglo pasado, el estudio de la traducción en el medio audiovisual ha tenido un gran crecimiento en España. Podemos verlo reflejado en las numerosas investigaciones llevadas a cabo. Entre los estudios más recientes encontramos los trabajos de Vulpoi (2018), Bolaños (2017), Botella (2017), Navarro (2017). Por supuesto, el mundo audiovisual ha variado mucho en las últimas décadas. Las posibilidades que ofrecen las nuevas tecnologías y plataformas digitales han cambiado la forma de consumo y la capacidad de decisión del público y con ello la situación del traductor en todo este proceso. Como explica Chaume (2018):

There's a exponential increase in today's active and passive consumption of audiovisual products, when compared to previous decades (Pérez-González, 2014). The global world media markets that produce audiovisual content and entertainment are absolutely aware of this and consequently, are becoming ever more aware of the role of translation, also referred to by the industry as localization, internationalization or even glocalization, that is, the process of adapting an audiovisual production to the norms, likes and dislikes of different target cultures.

Tomemos el ejemplo de la serie que analizamos: Stranger Things, producida por Netflix. En esta plataforma el consumidor puede elegir en segundos si quiere ver la película en versión original o doblada en español, francés, italiano o alemán, si desea la versión original audiodescrita o si incluir subtítulos. Otras plataformas similares son HBO o Amazon Prime Video. Todas ellas han impulsado en gran medida el sector de la traducción en España, pues el gran aumento de contenidos ha supuesto un incremento del volumen de trabajo. No obstante, cabe destacar que este fenómeno también ha repercutido en otros aspectos que afectan a la calidad del trabajo de los profesionales, pues la urgencia con la que se demandan los contenidos hace que los plazos sean cada vez más ajustados.

En el presente estudio nos centramos en una de las modalidades de traducción audiovisual, el doblaje, que consiste en la sustitución, en la banda sonora de un texto audiovisual, de la pista de diálogos original por una nueva pista con la versión grabada en lengua meta (De los Reyes, 2015).

En cuanto al papel del traductor, dentro de todo este proceso Marzà (2013) explica quiénes participan en él: 
El texto audiovisual pasa por las manos de productor, distribuidor, exhibidor, director del estudio de doblaje (para casting y producción), traductor, adaptador, actores, director y técnicos de doblaje antes de llegar al emisor final (Marzà, Torralba y Grupo TRAMA, 2013, p. 36).

Esta modalidad de traducción audiovisual también ha experimentado un notable crecimiento, pues la industria del entretenimiento requiere cada vez más, a través de las plataformas antes mencionadas, ofrecer productos audiovisuales de otros mercados (principalmente de Estados Unidos) y que para introducirse tanto en el mercado de España como en el de América Latina requieren contar con la opción de idioma español.

\section{LA TRADUCCIÓN DE LA VARIACIÓN LINGÜÍSTICA}

La variación lingüística es aquella que nos muestra la manera de hablar de una comunidad lingüística. Esta supone un gran reto para el traductor pues, como explica Tello (2012, p.143), se trata de un «artificio más en el abanico de herramientas estilísticas que enriquecen la obra» y que sirve para retratar los personajes y la sociedad donde se desarrolla la historia.

A la hora de abordar una traducción de un texto en el que se nos presenta una variación lingüística, sea cual sea su formato, debemos dilucidar cuál es la importancia y la finalidad del uso de dicha variante. Lomeña (2009, pp. 278-279) señala que existen diferentes opciones para la traducción de la variación lingüística, que deberemos elegir en función de diferentes factores extralingüísticos que afectarán a nuestra traducción. A saber:

La finalidad del uso de una variante, la información adicional que contenga, y otros elementos como el género de texto (si es audiovisual las restricciones en cuanto a estrategias serán mayores), el destinatario, la distancia entre la cultura origen y la cultura meta, los parámetros de aceptabilidad de esta última, etc., llegados a este punto no hay que olvidar que la traducción se concibe como un producto que debe cumplir ampliamente las expectativas de los destinatarios potenciales -sin los cuales el proceso traductológico carecería de sentido.

Esta autora también nos explica que existen «modalidades de traducción más sensibles a uno u otro canal como es el caso de la traducción audiovisual que por su carácter híbrido de oral-escrito plantea problemas relativos al modo o medio» (Lomeña, 2009, p. 279). 
La traducción de la variación lingüística en traducción audiovisual ha sido un tema recurrente en los estudios de traducción. A continuación haremos referencia a algunos de los aportes más recientes en los que se aborda la traducción de la variación lingüística en la modalidad audiovisual concreta del doblaje. Arampatzis (2011) en su tesis doctoral se centra en el estudio de la variación lingüística en la traducción de textos audiovisuales humorísticos a través del estudio de caso del doblaje al español de España de diversas escenas de las series Friends y Will y Grace. Su investigación aborda, sobre todo, la variación diatópica (los dialectos), la variación diastrática y la variedad mediática.

Uclés (2016) aborda la problemática de la traducción de la variación geográfica o diatópica a través de un corpus paralelo constituido por fragmentos de material audiovisual de ficción de habla inglesa y sus traducciones para el doblaje al español de España.

García (2018) aborda el doblaje del multilingüismo de la serie Jane the Virgin, adaptación de una telenovela que gira en torno a la vida de una familia de origen venezolano en Estados Unidos. Dicha autora se centra en el tratamiento de la variación lingüística en el discurso de los personajes, los insertos y las canciones tanto en la versión para España como para América Latina.

Por último, Zamora (2018) analiza el trasvase de las marcas lingüísticas temporales en la traducción al italiano de la novela El capitán Alatriste y en el doblaje de la película homónima. Para el presente estudio nos interesa sobre todo la parte de su trabajo en la que analiza las técnicas de traducción de la variación diacrónica en el doblaje. Las conclusiones de su investigación revelan una tendencia a la estandarización del lenguaje en la versión meta.

\subsection{La traducción de la variedad diacrónica}

Dentro de la variedad lingüística encontramos un tipo de variedad referida al tiempo, el espacio temporal en el que se ubica el texto objeto de nuestra traducción y que caracteriza la historia y los personajes. Esta se conoce como variedad diacrónica. Son varios los autores y autoras que han reflexionado sobre las estrategias de traducción a la hora de trasvasar esta variedad. Catford (1965) habla de dialectos temporales arcaicos y señala que no hay que buscar una equivalencia total al traducirlos, pero sí se puede plasmar ese "sabor arcaico» (a través de marcadores léxicos o fonológicos); mientras que Rabadán (1991) recomienda no traducir los rasgos temporales, ya que el texto estará marcado por otros indicadores característicos del estadio diacrónico. Más adelante, Hatim y Mason (1995 
p. 60) señalan que los dialectos temporales suponen tanto una dificultad para los traductores (al tener que estar al corriente de cada cambio que acontezca en la lengua) como un problema de compresión incluso para el público original. Proponen, bien traducir hacia una variante arcaica de la lengua meta, o bien hacia la variante contemporánea. Por último, Muñoz (1995) afirma que incluso si queremos traducir intentando reproducir la intención del autor, la lectura estará mediatizada por la perspectiva contemporánea del traductor y del público a quien está destinada la traducción. Por lo tanto, a la hora de traducir la variación diacrónica el traductor se debatirá entre el uso de una estrategia modernizante, neutralizando la mayor parte de las marcas diacrónicas presentes en el texto original o por una estrategia arcaizante, en la que intente preservar la mayor parte de esos rasgos. Asimismo, se podrá optar por una estrategia intermedia en la que la variación diacrónica se mantenga parcialmente (Zamora, 2018).

En el caso concreto que analizamos en el presente estudio, la dificultad no radica en el alejamiento en el tiempo del original y la traducción, sino en cómo reproducir el lenguaje marcado en diacronía que los autores del producto original han empleado para enmarcar la época en la que se ambienta la serie (años 80 ). Por lo tanto, se trata de un producto actual ambientado en una época y cultura más antigua. A continuación nos centraremos en los elementos que permiten definir la variedad diacrónica en la serie objeto de estudio. Cabe destacar que dado que los protagonistas de la serie son adolescentes nos centraremos principalmente en el lenguaje juvenil, un tipo de habla que se caracteriza por «la acepción de ciertas formas subestándar como medio de distinguirse de la lengua estándar» (Rodríguez, 2006).

Rodríguez (2006) identifica cuatro tipos de recursos expresivos recurrentes que caracterizan los sociolectos juveniles. Reproduciremos a continuación los que nos interesan desde el punto de vista del lenguaje oral que será el que se transmita en el doblaje de la serie:

a) Cambio de significante que puede llevarse a cabo mediante el denominado truncamiento (acortamiento de palabras), como por ejemplo estar depre (deprimido) o también mediante la segmentación de palabras, como en el caso de masoca (masoquista). Asimismo se aprecia el uso de sufijos (-ata, -eta, -ota, -aca) con una función connotativa (drogata> drogadicto). 
b) Cambio semántico: creación de palabras nuevas o empleo de las ya existentes con un significado diferente. Por ejemplo uso del término tarro como sinónimo de cabeza.

c) Cambio de código: uso de palabras o expresiones ya existentes, procedentes de otros sociolectos (parné como sinónimo de dinero o trena como sinónimo de cárcel).

d) Cambio de registro: uso de vulgarismos o expresiones informales como marcadores de expresividad (pelas en lugar de dinero, tener jeta por tener mucha cara).

A continuación exponemos los rasgos más característicos del lenguaje de los ochenta en las dos sociedades objeto de estudio en el presente artículo:

2.1.1. El lenguaje juvenil de los ochenta en la sociedad estadounidense.

El lenguaje de la juventud estadounidense de la década de los ochenta presenta, entre otros, los siguientes rasgos:

- Se forman nuevas palabras que surgen para cubrir los vacíos semánticos existentes y que reflejan las preocupaciones de esa década por la comida saludable, el medioambiente o la economía, entre otras áreas. Por ejemplo: ozone-friendly, greenhouse effect.

- A partir de palabras ya existentes, estas ganan nuevos significados para referirse a los nuevos conceptos que surgen en la sociedad.

- Adquisición de palabras propias de culturas que se consideran un referente entre la juventud (como la cultura californiana surfera).

- Nuevas palabras con la adición de prefijos. Por ejemplo el prefijo -dis, utilizado en la palabra disrespect pasó a usarse en el lenguaje informal.

2.1.2. El lenguaje juvenil de los ochenta en la sociedad española.

Los rasgos lingüísticos juveniles en España suelen estar marcados por connotaciones del léxico marginal y juvenil, que guarda relación con la época histórica en la que se desarrollan en el país. Rodríguez (1989) destaca, entre otros, los siguientes: 
- Deformar el significante, por mutilación o alteración de su morfología.

- Nueva relación entre el significante y significado (supone una gran polisemia).

- Trisilabismo, abreviar nociones y palabras largas y formar una palabra de tres sílabas (ecologista>ecolo; manifestación>manifa).

- Vocabulario con origen en el léxico de la droga (movida, muermo, alucinar) u otras como bocata, cubata, taco (año) y kilo (millón pesetas).

- Préstamos, sobre todo del inglés (look).

\section{Metodología}

El objetivo del presente trabajo es comprobar qué estrategias de traducción se han seguido a la hora de trasvasar la variación diacrónica, es decir, si esta se mantiene totalmente, si se mantiene parcialmente o si no se mantiene. Para ello analizaremos también qué técnicas de traducción se han empleado siguiendo la clasificación de Martí (2007). Su propuesta se basa en el estudio de las contribuciones de numerosos académicos de la traductología. Las técnicas de traducción nos trasmiten las soluciones que toma el traductor para resolver los problemas microtextuales. Estas son las 20 técnicas de traducción que presenta Martí ordenadas en función del método de traducción, siendo 1 la más cercana al método literal y la 20 la más cercana al método interpretativo-comunicativo:
1. Préstamo
2. Calco
3. Traducción palabra por palabra
4. Traducción uno por uno
5. Traducción literal
6. Equivalente acuñado
7. Omisión
8. Reducción
9. Compresión
10. Particularización
11. Generalización
12. Transposición
13. Descripción
14. Ampliación
15. Amplificación 


\author{
16. Modulación \\ 17. Variación \\ 18. Sustitución \\ 19. Adaptación \\ 20. Creación discursiva
}

De este objetivo surgen dos hipótesis:

Por un lado, consideramos que no será posible trasladar todos los elementos característicos del lenguaje de los años ochenta, ya sea por falta de equivalentes en la cultura meta o por las limitaciones que supone el código de colocación de sonido en la traducción audiovisual para el doblaje.

Por otro lado, suponemos que en los casos en los que no sea posible trasladar la referencia de la variedad diacrónica, la tendencia traductora será utilizar un término más general o neutro, que no tenga una marca diacrónica pero que siga respetando el texto original.

La aproximación metodológica del presente artículo parte de la lingüística de corpus, una rama de la lingüística que se ocupa de la realización de «análisis lingüísticos léxicos, gramaticales, semánticos y pragmáticos (discursivos) mediante el uso de herramientas informáticas diseñadas para este fin» (Bolaños 2015: 34).

La base metodológica de nuestro proyecto consta de varias fases.

En primer lugar cabe hablar de los criterios de selección de la obra objeto de estudio. Consideramos que se trata de una serie de especial interés dado su enorme éxito tanto en Estados Unidos como en España, como así queda patente en el informe de Parrot Analytics sobre la demanda televisiva en el año 2017 y en el que se indica que Stranger Things fue la serie más vista en España a lo largo de dicho año. Asimismo, se trata de una serie contextualizada en los años ochenta, época que no solo se retrata a través de los decorados o la escenografía, sino que también aparece reflejada en ciertas intervenciones de los personajes. Por lo tanto, el estudio de esta obra nos parece relevante a la hora de abordar la traducción de la variación diacrónica.

La segunda fase se corresponde con la compilación de un corpus paralelo a partir de la transcripción de los guiones de dos temporadas de la serie Stranger Things.

En tercer lugar procedimos a realizar la selección de elementos característicos del inglés en los años ochenta en Estados Unidos. Para comprobar que efectivamente estos términos 0 expresiones se correspondían con la época en la que se enmarca la historia recurrimos principalmente a dos corpus:

- Corpus of Contemporary American English (COCA)

- Corpus of Historical American English (COHA) 
Estas dos fuentes nos sirvieron para detectar el léxico de los años ochenta en inglés, así como el crecimiento y decrecimiento del uso de dichos términos y expresiones.

A continuación pasamos a analizar si el léxico elegido en la traducción al español también se correspondía con la época objeto de estudio. Para ello recurrimos también a dos corpus:

- Corpus diacrónico del español (CORDE)

- Corpus de referencia del español actual (CREA)

Asimismo en ambas lenguas usamos la herramienta NgramViewer a modo de corpus de contraste, para corroborar la veracidad de los datos que nos ofrecían los corpus.

Con el fin de comprobar el significado de muchos de los términos y expresiones tanto en inglés como en español y verificar que el sentido del texto meta se correspondía con el del texto original utilizamos varios diccionarios disponibles en línea. Uno de los más útiles fue el diccionario Merrian Webster, pues cuenta con una sección llamada First Known Use que nos ofrecía información sobre la primera vez que un determinado término se usó. Entre los demás diccionarios utilizados destacan American Heritage Dictionary, Wordnik, Oxford English Dictionaries, UrbanDictionary y The Free Dictionary.

Por último, cabe destacar que dada la formalidad de muchas de estas obras lexicográficas y la informalidad o coloquialidad del lenguaje objeto de análisis en el presente artículo, en ocasiones ha sido necesario utilizar fuentes menos conocidas y de menor fiabilidad como páginas o blogs como parte de nuestro proceso de documentación, pues en muchos de los recursos analizados no siempre aparecía recogido el lenguaje coloquial, en ocasiones vulgar y con la variedad diacrónica que nos ocupa en este trabajo.

\subsection{Corpus del trabajo}

En este apartado presentamos el producto analizado, la serie de Netflix Stranger Things.

La serie se desarrolla en el pueblo de Hawkins en Estados Unidos a mediados de los años ochenta. Un niño del pueblo, Bill Byers, desaparece. Su familia y amigos lo buscan y empiezan a ocurrir sucesos extraños. Se trata de un producto audiovisual cargado de referencias a la cultura y sociedad estadounidense de la época, así como de expresiones, argot y vocabulario característicos de entonces.

A continuación, mostramos la ficha técnica de la serie:

\section{STRANGER THINGS}

Hikma 18 (1) (2019), 315 - 345 


\begin{tabular}{|c|c|}
\hline Directores & Matt y Ross Duffer \\
\hline Género & $\begin{array}{ll} & \text { Series TV juveniles } \\
\text { Thrillers TV } & \\
\text { Ciencia ficción y fantásticas de }\end{array}$ \\
\hline Fechas de estreno & $\begin{array}{l}15 \text { de julio de } 2016 \text { y } 27 \text { de } \\
\text { octubre de } 2017\end{array}$ \\
\hline Año de grabación & 2016 y 2017 \\
\hline Director de doblaje & Ramón Hernández \\
\hline Traductora & Glòria Drugis \\
\hline Ajustadora & Glòria Drugis \\
\hline Estudio de grabación & $\begin{array}{ccc}\text { Deluxe } & \text { Spain } & \text { (Madrid, } \\
\text { Barcelona) } & & \end{array}$ \\
\hline Distribuidora para España & Netflix España \\
\hline Distribuidora original & Netflix \\
\hline Productora & 21 LAPS ENTERTAINMENT \\
\hline Capítulos analizados & $\begin{array}{l}\text { Temporada 1: } \\
\text { 1. La desaparición de Will Byers } \\
\text { 2. La chica rara de la calle } \\
\text { Maple } \\
\text { 3. Luces navideñas } \\
\text { 4. El cadáver } \\
\text { 5. La pulga y el acróbata } \\
\text { 6. El monstruo } \\
\text { 7. La bañera } \\
\text { 8. Del revés } \\
\\
\text { Temporada 2: } \\
\text { 1. MADMAX } \\
\text { 2. Truco o trato, bicho raro } \\
\text { 3. El renacuajo } \\
\text { 4. Will el Sabio } \\
\text { 5. Digdug }\end{array}$ \\
\hline
\end{tabular}

Hikma 18 (1) (2019), 315 - 345 


\begin{tabular}{|l|l|}
\hline & 6. El espía \\
& 7. La hermana perdida \\
& 8. El azotamentes \\
& 9. El portal \\
\hline
\end{tabular}

Para llevar a cabo el análisis de corpus se han organizado los elementos característicos del lenguaje de los ochenta en diferentes categorías. En total hemos detectado 5 categorías que se han denominado del siguiente modo:

1. Expresiones coloquiales

2. Vocabulario específico de los ochenta

3. Contracciones

4. Expresiones soeces

5. Derivación

\section{ANÁLISIS}

A continuación pasaremos a analizar las expresiones extraídas de la primera y segunda temporada de la serie que consideremos características de los años ochenta. En total hemos detectado 23 ejemplos en inglés de dicha época en Estados Unidos, de los cuales 6 pertenecen a la categoría de expresiones coloquiales, 3 a la de vocabulario específico de los 80, 3 a la de contracciones, 9 a la de expresiones soeces y 1 a la derivación. En general, 13 de los casos han mantenido la variedad diacrónica en la versión doblada en español, 2 lo hacen parcialmente y 8 no la mantienen. 


\section{3 expresiones analizadas}

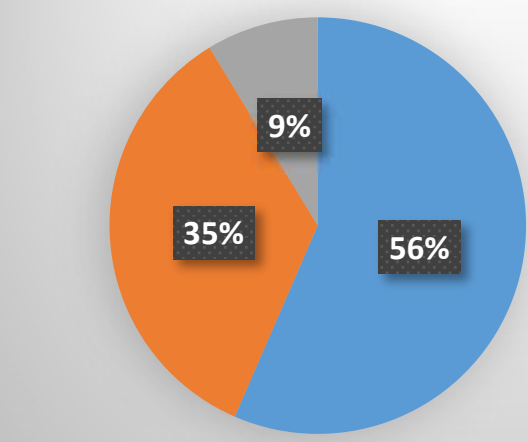

Mantienen la

variedad diacrónica.

No mantienen la variedad diacrónica

Mantiene

parcialmente la variedad diacrónica.

\section{Expresiones por categorías}

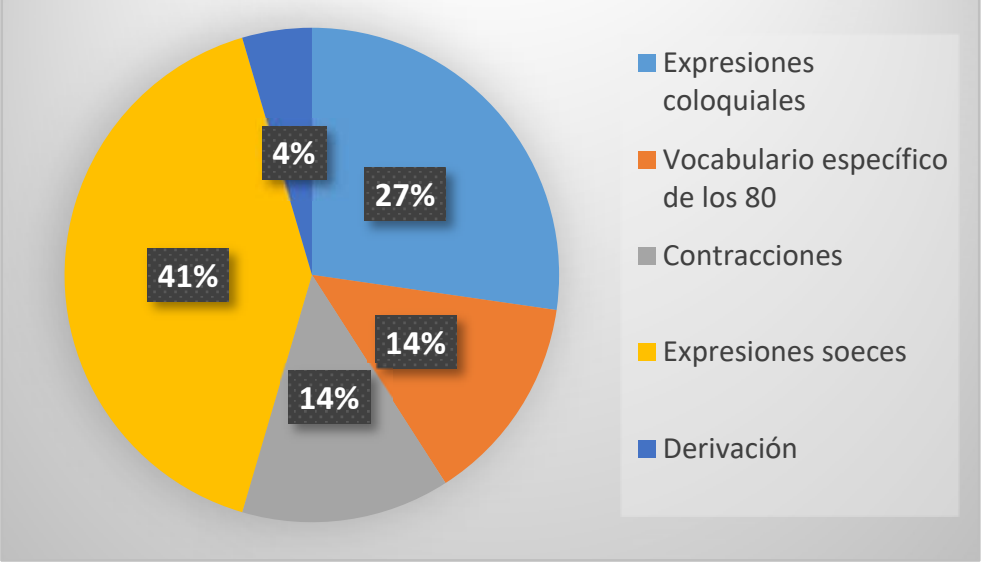

Para nuestro análisis proporcionaremos algunos de estos términos o expresiones acompañados de una definición y contextualización, organizados en categorías. Observaremos cuál ha sido su traducción al español y evaluaremos su contexto, vigencia y limitaciones.

Hikma 18 (1) (2019), 315 - 345 
Los ejemplos se presentan en formato de tabla en la que incluimos el número de la temporada (T) y capítulo (C), así como el nombre del personaje que pronuncia la expresión y la técnica y estrategia de traducción utilizadas.

\subsection{Expresiones coloquiales}

Ejemplo 1: Cool

\begin{tabular}{|c|c|}
\hline \multicolumn{2}{|c|}{ T1C1 - Barbara } \\
\hline $\begin{array}{l}\text { Nance, seriously. You're } \\
\text { going to be so cool now it's } \\
\text { ridiculous. }\end{array}$ & $\begin{array}{c}\text { Nancy, vamos. Ahora vas a } \\
\text { ser tan guay que ni te lo creerás. }\end{array}$ \\
\hline Técnica de traducción & Equivalente acuñado \\
\hline Estrategia de traducción & Mantiene la variedad diacrónica \\
\hline
\end{tabular}

Tabla 1: Cool

Esta palabra en inglés tiene multitud de acepciones. En este caso, como indica Kelly (2016) "By 1983, cool, both as "hip" and "OK," was wellsettled into the teenage lexicon. Beyond Barb, Stranger Things uses cool freely-and appropriately so-throughout the series».

La acepción que recoge el diccionario Merriam Webster (2018) más cercana a nuestro contexto es «very good, excellent. Also: allright».

Dada la polisemia de la palabra, resulta bastante complicado determinar mediante corpus el uso de la misma con este sentido en la época de los ochenta; sin embargo, tenemos diversas páginas que hablan sobre el vocabulario de la época, como NgramViewer, que corroboran que era característica de entonces.

En el caso de la traducción española, encontramos la palabra en la década de los ochenta en NgramViewer, no así en el CREA, que la registra a partir de los noventa. Esto se puede deber al problema que ya mencionamos anteriormente, es vocabulario coloquial y más propio del lenguaje oral, por lo que es menos probable que este quede registrado en los corpus. La consulta con NgramViewer muestra un crecimiento del uso a partir de la década de los 80, donde pasa del $0 \%$ en 1975 al $0,000000074 \%$ en 1982 . En definitiva, es una traducción que respeta la temporalidad y registro del original. 
Respecto a la imagen y la isocronía, pese a que el personaje pronuncia la expresión en un primer plano, esto no ha supuesto una limitación para la traducción como en otros casos. La expresión original y su traducción tienen una extensión parecida y además, la boca del personaje aparece tapada varias veces durante el diálogo.

Ejemplo 2: Lowkey

\begin{tabular}{|l|l|}
\hline \multicolumn{2}{|l|}{ T1C2 - Steve } \\
\hline It'll be low key. & Será tranquila. \\
\hline Técnica de traducción & Generalización \\
\hline Estrategia de traducción & Mantiene la variedad diacrónica \\
\hline
\end{tabular}

\section{Tabla 2: Lowkey}

Esta expresión puede usarse en inglés para definir algo que es secreto o que no es muy complejo, intenso o excesivo, sino más bien modesto y tranquilo.

Como podemos observar en NgramViewer, esta expresión tiene un uso bastante anterior a la década de los ochenta pero es a partir de los años setenta cuando su uso crece notablemente (de 0'00000513 \% en 1960 a $0.0000154 \%$ en 1980). Lo mismo encontramos en el COHA, donde hay un mayor número de ejemplos entre la década de los 70 y los 90 . De un total de 67 ejemplos desde principios del siglo XIX, 13 aparecen en los 70, 10 en los 80 y 9 en los 90 .

En este caso, la traducción al español traslada el sentido del mensaje original, pero no presenta ningún rasgo característico de los años ochenta.

El personaje que pronuncia esta expresión se encuentra de lado durante la escena y la imagen de la boca no está muy visible, por lo que la imagen no nos dificulta la traducción en este caso.

Ejemplo 3: Buzz-cut or buzz cut

\begin{tabular}{|c|c|}
\hline \multicolumn{2}{|l|}{ T1C2 - Hopper } \\
\hline If this kid had a buzz cut. & $\begin{array}{l}\text { Si este chaval se rapara el } \\
\text { pelo. }\end{array}$ \\
\hline Técnica de traducción & Transposición \\
\hline Estrategia de traducción & No mantiene la variedad \\
\hline
\end{tabular}

Hikma 18 (1) (2019), $315-345$ 


\begin{tabular}{|l|l|}
\hline & diacrónica \\
\hline
\end{tabular}

Tabla 3: Buzzcut

El término buzz-cut o buzzcut, se refiere a un tipo de peinado con el pelo rapado.

Según el diccionario Merriam Webster (2017) este es «a very short haircut usually for men or boys in which the hair resembles the bristle surface of a brush».

La primera vez que este diccionario tiene registrado su uso es en 1977. Sin embargo, si consultamos el corpus de Google, NgramViewer, vemos que este término aparece a partir de 1985, manteniendo un uso muy bajo hasta 1990 (0,000000225 \%) y únicamente aparece sin guion.

La traducción al español, al no existir un término equivalente en español para esa época, utiliza una técnica de transposición en la que se cambia la categoría gramatical y se hace una generalización, de usar un sustantivo para el peinado a explicar la acción que le lleva a tener ese peinado, raparse el pelo. En cuanto a la imagen y las restricciones que supone para la traducción para el doblaje, debemos tener en cuenta que se trata de un primer plano, como mostramos a continuación, por lo que la traducción está limitada por la isocronía y la sincronía.

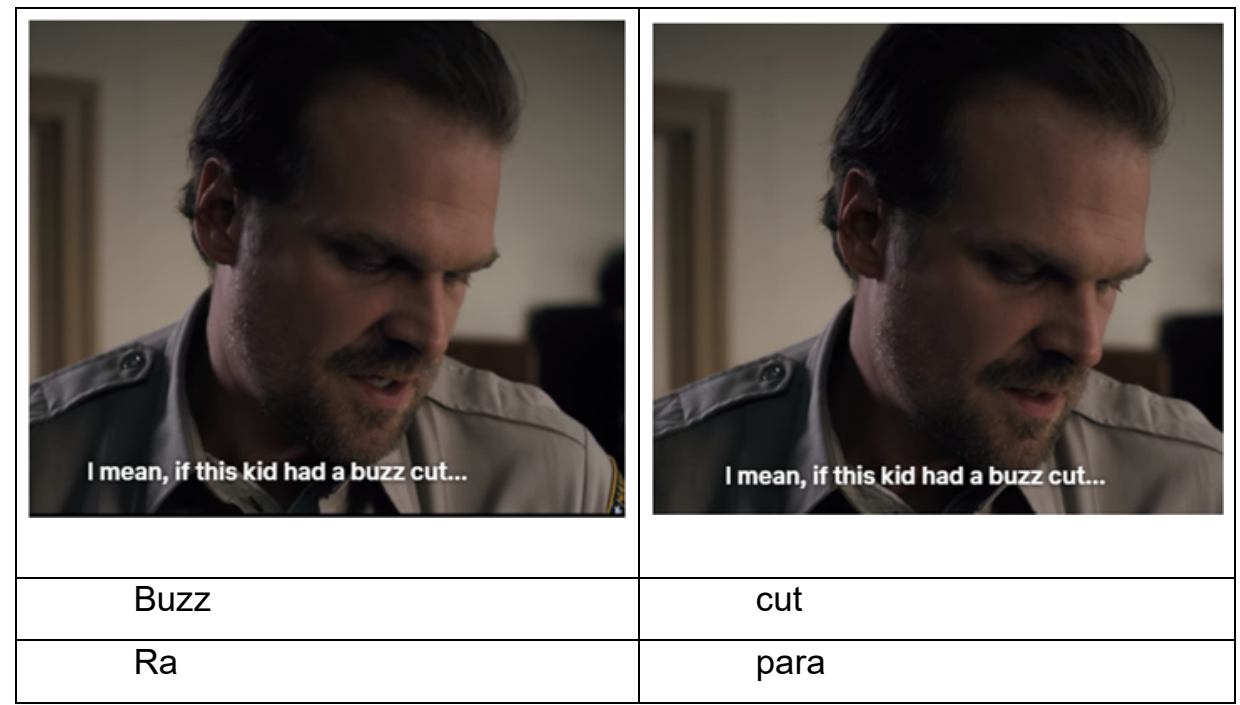

Hikma 18 (1) (2019), $315-345$ 
Ejemplo 4: Gnarly

\begin{tabular}{|l|l|}
\hline \multicolumn{2}{|l|}{ T1C2 - Tommy } \\
\hline Gnarly! & ¡Qué fuerte! \\
\hline Técnica de traducción & Generalización \\
\hline Estrategia de traducción & No mantiene la variedad \\
& diacrónica \\
\hline
\end{tabular}

Tabla 4: Gnarly

Esta expresión es característica del lenguaje coloquial en los años ochenta. Procede de la cultura surfera de la zona de California que durante esta década se extendió por Estados Unidos, variando en ocasiones su significado, como explica Soony (2009) en su blog:

Originally, gnarly was applied to something that was tough or a bit ragged, particularly a set of towering waves or a skateboard trick gone wrong. "Did you see his arm after he ate it hard on that halfpipe? Gnarly, man." Gradually, however, it took on a new meaning as a way to describe something good. "Gnarly pad you've got man. I'm totally stoked for tonight's party." Today, the word gnarly lives on, and can bear either meaning depending upon the situation.

En esta ocasión la traducción al español utiliza una expresión más neutra, que encaja en el contexto, pero pierde el matiz de la época de los ochenta que sí tiene la expresión original. NgramViewer nos muestra que esta expresión está presente en los 80 , pero lo está igualmente desde principios de siglo. A partir de 1985 aumenta considerablemente su uso (1985: 0.0000020\%, 2000: 0,00000976 \%. El COHA también nos muestra un aumento de su uso, pero a partir de 1990 (de 86 ejemplos desde principios del siglo XIX hay 5 en la década de los 80,23 en las de los 90 y 18 en los 2000).

De nuevo, tenemos que tener en cuenta las restricciones del doblaje. Como veremos en la siguiente tabla, en este caso también se trata de un primer plano y se adelanta la intervención en la versión español para que esta se ajuste la imagen. 


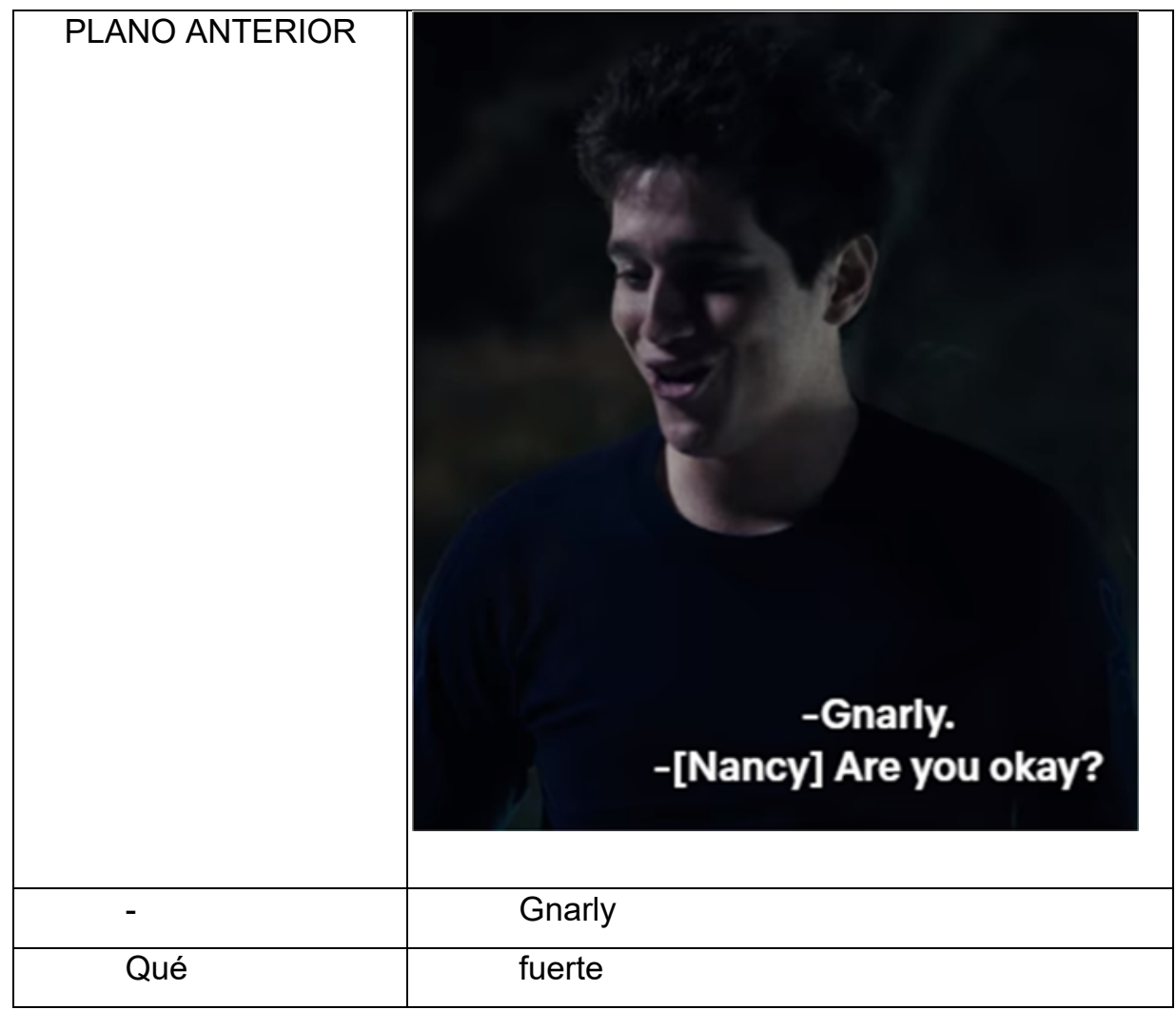

4.2. Vocabulario específico de los 80

Ejemplo 5: Tubular

\begin{tabular}{|l|l|}
\hline \multicolumn{2}{|l|}{ T2C2 - Dustin } \\
\hline Yeah, totally tubular. & Es super tubular. \\
\hline Técnica de traducción & No mantiene la variedad \\
\hline Estrategia de traducción & diacrónica \\
\hline
\end{tabular}

Tabla 5: Tubular

Hikma 18 (1) (2019), 315 - 345 
Tubular, con la acepción de este contexto, no aparece definido en diccionarios como Merriam Webster, Word Reference o AHdictionary, pero sí podemos verlo en Oxford Dictionary (2018), que indica que es propio del inglés estadounidense, informal y se refiere a algo "wonderfuloramazing».

Como nos indica el blog de Oxford Dictionaries (2017) en su guía sobre el lenguaje de Stranger Things, se usaba en la época de los ochenta para definir algo impresionante, increíble, como la ola perfecta para surfear, donde tiene su origen la palabra. Esta expresión es parte del argot conocido como Valley slang, que se popularizó en la cultura estadounidense de los años ochenta.

En el contexto de la serie, se trata de una palabra muy novedosa y moderna para Lucas y Dustin, que tratan de usarla igual que Max, cuyo lugar de origen, California, es de donde proviene la expresión. No encontramos registro de esta expresión en los corpus COHA o NgramViewer y solo el COCA recoge dos ejemplos de la palabra que coinciden con el contexto que nos ocupa, pero del año 2012, muy posteriores a la época de la serie.

En el caso de la palabra en español, observamos que ni el Diccionario de la Real Academia Española ni el corpus CREA recogen ejemplos con esta acepción. Por tanto, vemos que en la traducción se ha recurrido al uso del préstamo y se mantiene un término típico de la cultura estadounidense de los ochenta que desconocemos en la cultura meta. No obstante, debemos tener en cuenta que la imagen en este ejemplo, vuelve a ser un primer plano, en el que además se repite la expresión varias veces seguidas y se marca la pronunciación. Esto limita enormemente la labor de traducción:

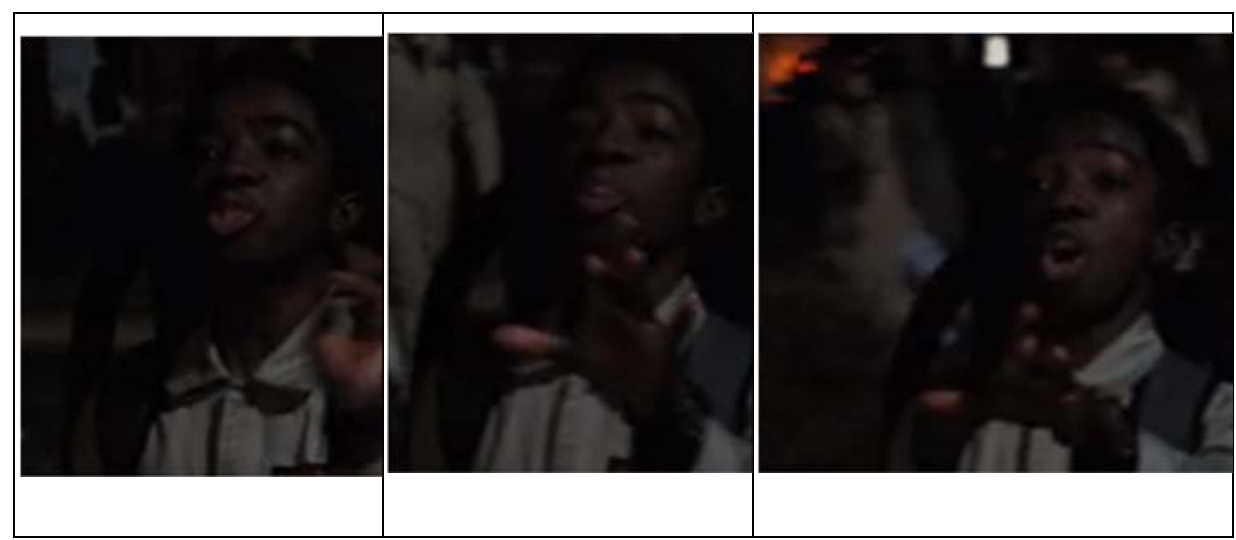

Hikma 18 (1) (2019), 315 - 345 


\begin{tabular}{|c|c|c|}
\hline Tu & bu & lar \\
\hline Tu & bu & lar \\
\hline
\end{tabular}

Ejemplo 6: Drunk as a skunk

\begin{tabular}{|c|c|}
\hline \multicolumn{2}{|l|}{ T1C2 - Callahan } \\
\hline And he was drunk as a skunk. & Estaba como una cuba. \\
\hline Técnica de traducción & Equivalente acuñado \\
\hline Estrategia de traducción & Mantiene parcialmente la \\
\hline
\end{tabular}

Tabla 6: Drunk as a skunk

Esta expresión se utiliza para expresar de forma coloquial que una persona está muy borracha. Su uso aparece registrado tanto en NgramViewer como en el COHA desde la década de los cuarenta pero es a partir de la década de los setenta cuando aumenta notablemente su uso. En 1970 NgramViewer mostraba un uso del 0'000000438 \%, mientras que en 1990 llega al 0,00000592\%.

En el caso de la traducción elegida, «estar como una cuba», se trata de una expresión muy extendida en la cultura española para definir el mismo concepto de forma coloquial. En este caso, su uso se registra desde principios del siglo $\mathrm{XX}$ y se mantiene de forma constante el uso (aproximadamente un 0,000002 \%) hasta finales de la década de los 80 , cuando comienza aumentar hasta el 0,000004 \% en 2000.

En este caso la imagen no afecta en absoluto a la traducción, ya que el personaje que habla está de espaldas en ese momento.

\subsection{Contracciones}

Ejemplo 7: Told ya

\begin{tabular}{|l|l|}
\hline \multicolumn{2}{|l|}{ T1C1 - Dustin } \\
\hline Told ya. & ¿Lo ves? \\
\hline Técnica de traducción & Generalización \\
\hline
\end{tabular}

Hikma 18 (1) (2019), 315 - 345 


\begin{tabular}{|l|l|}
\hline Estrategia de traducción & No mantiene la variedad diacrónica \\
\hline
\end{tabular}

Se trata de una forma más coloquial de I told you. Podemos ver que esta expresión aparece en el COHA a partir de los años cuarenta, pero es en los ochenta donde se registra el mayor uso. De los 41 ejemplos que nos ofrece entre 1960 y 2000, 12 pertenecen a la década de los 80. Sin embargo, en NgramViewer el uso de la expresión es notable en los años ochenta, donde llega a alcanzar un uso de $0,00000288 \%$ pero sigue ascendiendo en las siguientes décadas.

La traducción literal al español sería «te lo dije», la cual no tiene ninguna marca temporal que nos traslade a los años ochenta y supone un problema de sincronización, ya que tiene el doble de sílabas que el original. La opción elegida para la versión española es "¿Lo ves?», una expresión que mantiene un sentido similar al original y que soluciona el problema de la isocronía, así como el de la sincronía fonética, pues la articulación bucal de «told ya» es similar a la de «¿lo ves?», como mostramos a continuación:

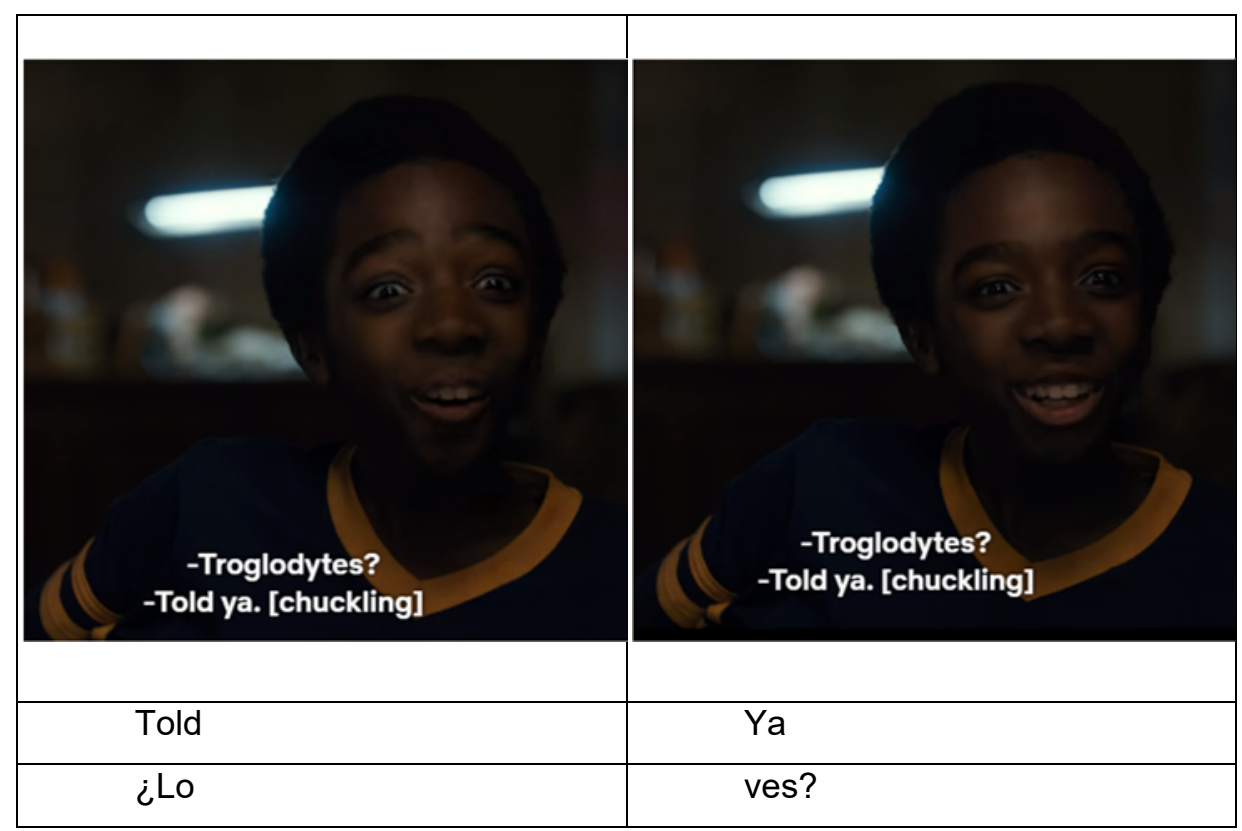

Hikma 18 (1) (2019), 315 - 345 
Ejemplo 8: I think it's kinda cool

\begin{tabular}{|l|c|}
\hline \multicolumn{2}{|l|}{ T1C1 - Mike } \\
\hline I think it's kinda cool. & Yo creo que es una pasada. \\
\hline Técnica de traducción & Equivalente acuñado \\
\hline Estrategia de traducción & Mantiene la variedad diacrónica \\
\hline
\end{tabular}

Tabla 8: It's kinda cool

En relación con el ejemplo anterior, encontramos también otra expresión característica del lenguaje oral en Estados Unidos. Se trata de una contracción de it's kind of cool y según podemos ver en NgramViewer este término se usó en los años ochenta y a partir de esa década aumenta notablemente el registro de su uso. Utilizamos la misma herramienta para analizar el término usado en la traducción española y observamos que coinciden, tanto en el uso durante esa década como en el ascenso en su uso a partir de entonces. Vemos, por tanto, que en este caso se trata de una traducción que mantiene tanto el matiz coloquial como el temporal.

En este caso la imagen no afecta en absoluto a la traducción, ya que el personaje que habla está de espaldas en ese momento.

\subsection{Expresiones coloquiales}

\section{Ejemplo 9: Mouth-breather}

\begin{tabular}{|c|c|}
\hline \multicolumn{2}{|l|}{ T1C3 - Mike } \\
\hline $\begin{array}{l}\text { - I was tripped by this mouth- } \\
\text { breather, Troy, okay? }\end{array}$ & $\begin{array}{l}\text { - Me ha hecho la zancadilla } \\
\text { un abusón. Troy, ¿vale? }\end{array}$ \\
\hline - $\quad$ Mouth-breather? & - ¿ ¿Un abusón? \\
\hline $\begin{array}{l}\text { - Yeah, you know a dumb } \\
\text { person. A knucklehead. }\end{array}$ & $\begin{array}{l}\text { - Sí, ya sabes un idiota, un } \\
\text { cabeza hueca. }\end{array}$ \\
\hline Técnica de traducción & Sustitución \\
\hline Estrategia de traducción & No mantiene la variedad diacrónica \\
\hline
\end{tabular}

Tabla 9: Mouth-breather

Como indica Oxford Dictionaries en su blog, mouth-breather fue en su origen un término exclusivamente médico que describía a aquellas personas 
que respiraban por la boca y así fue hasta los años setenta. A partir de entonces los jóvenes estadounidenses lo empezaron a usar como un insulto para llamar a alguien idiota, tonto, o retrasado (Oxford Dictionaries, 2017).

Curiosamente, si buscamos esta palabra en NgramViewer observamos que su uso es mucho mayor a principios del siglo $X X$ y va decayendo hasta nuestra época. Esto se debe probablemente a que nos muestra con el uso del término médico y hay un registro mucho menor de esta palabra como insulto. Al buscar la palabra escrita junta y sin guion, mouthbreather, encontramos que sigue el mismo patrón pero tiene un repunte en el uso entre los 70 y los $90(0,000000055 \%$ en 1970 , $0,000000137 \%$ en 1982 , el máximo, y $0,000000105 \%$ en 1990). Esto nos hace pensar que aunque el término médico se escriba con guion, la palabra como insulto se escribía más a menudo sin él.

Podemos encontrarlo en entradas en varias entradas de blogs de diccionarios como el Oxford English Dictionary o el Collins English Dictionary que tratan sobre el lenguaje de los ochenta en la serie.

En cuanto a la traducción en español, vemos que supone un pequeño cambio del sentido original, pues se usa como un insulto para llamar idiota o tonto a Troy, como después explica a Once. El término elegido es abusón, el cual no concuerda con el insulto original, pero sí encaja en el contexto puesto que Troy sí es un abusón. Posteriormente veremos a Once utilizar este término en otras escenas, que usa para describir a personas que considera malas. Este término es común en la época que nos ocupa, pero también en las décadas anteriores y posteriores, por lo que no es característico de los años ochenta.

En este ejemplo la imagen no nos muestra la boca del locutor en primer plano, si no que lo vemos de lado y en movimiento. Por tanto, la isocronía no dificulta la traducción, si bien sí tendremos que tener en cuenta la sincronía.

\subsection{Derivación}

Ejemplo 10: Weirdo

\begin{tabular}{|l|l|}
\hline \multicolumn{2}{|l|}{ T1C2 - Título del capítulo } \\
\hline The weirdo on Mapple Street. & $\begin{array}{c}\text { La chica rara de la calle } \\
\text { Mapple. }\end{array}$ \\
\hline Técnica de traducción & Equivalente acuñado \\
\hline
\end{tabular}

Hikma 18 (1) (2019), $315-345$ 


\begin{tabular}{|l|l|}
\hline Estrategia de traducción & $\begin{array}{c}\text { Mantiene la variedad } \\
\text { diacrónica }\end{array}$ \\
\hline
\end{tabular}

\begin{tabular}{|l|l|}
\hline \multicolumn{2}{|l|}{ T1C2 - Lucas } \\
\hline What is the weirdo doing? & ¿Qué hace la pirada? \\
\hline Técnica de traducción & Equivalente acuñado \\
\hline Estrategia de traducción & $\begin{array}{c}\text { Mantiene la variedad } \\
\text { diacrónica }\end{array}$ \\
\hline
\end{tabular}

\begin{tabular}{|c|l|}
\hline \multicolumn{2}{|c|}{ T1C3 - Lucas } \\
\hline $\begin{array}{c}\text { You seriously think that the } \\
\text { weirdo knows where Will is? }\end{array}$ & $\begin{array}{c}\text { ¿En serio crees que el bicho } \\
\text { raro sabe dónde está Will? }\end{array}$ \\
\hline Técnica de traducción & Equivalente acuñado \\
\hline Estrategia de traducción & Mantiene la variedad \\
& diacrónica \\
\hline
\end{tabular}

\begin{tabular}{|c|c|}
\hline \multicolumn{2}{|c|}{ T1C5 - Dustin } \\
\hline $\begin{array}{c}\text { You're asking if the weirdo is } \\
\text { acting weird? }\end{array}$ & $\begin{array}{c}\text { ¿Me preguntas si la rarita está } \\
\text { muy rara? }\end{array}$ \\
\hline Técnica de traducción & Equivalente acuñado \\
\hline Estrategia de traducción & Mantiene la variedad \\
\hline
\end{tabular}

Tabla 10: Weirdo

Weirdo aparece repetidamente a lo largo de la serie, ya que es el apodo que Lucas usa de forma despectiva para referirse a Once hasta el capítulo 6 de la primera temporada.

El Merriam Webster (2018) data su primera aparición en 1955 y se refiere a «a person who is extraordinarily strange or eccentric». 
NgramViewer nos muestra un uso extendido de la palabra en la década de los ochenta y su uso sigue creciendo hasta nuestros días. En el COHA podemos ver que hay un mayor uso de esta palabra en la década de los ochenta y los noventa. De 77 ejemplos mostrados, 25 pertenecen a los 80 y 30 a los 90 .

Como podemos observar en los cuatro ejemplos, en la traducción al español no usan un solo término, sino que encontramos cuatro diferentes: la chica rara, pirada, bicho raro y rarita. Todas se repiten durante los primeros seis capítulos, pero «bicho raro» es la expresión que más aparece de las cuatro. Consideramos que esta variación se debe principalmente a la limitación por la isocronía, como en el tercer ejemplo, donde dice "pirada» en lugar de «bicho raro» para adaptarse a la longitud del parlamento. En NgramViewer el uso de la expresión «bicho raro» crece del $0 \%$ hasta el 0,000000023\% en 1990. Todos los términos usados aparecen con frecuencia durante la década de los ochenta y mantienen el carácter coloquial y despectivo que tiene weirdo en el original.

\section{CONCLUSIONES}

Al inicio de este estudio nos planteábamos dos hipótesis. Una vez hecho el análisis, constatamos que ambas se han cumplido, pues no se han podido trasladar todos los elementos característicos de la variedad diacrónica y cuando esto no ha sido posible la tendencia ha sido a neutralizar los términos, sin modificar el mensaje original. Así, de los 23 ejemplos en inglés que hemos analizado, encontramos que la traducción 13 de ellos, es decir, un $56,52 \%$, se consigue el sentido, registro, intención y variedad diacrónica del elemento original. De los 10 ejemplos restantes, 8 , el $34,78 \%$, no mantienen la variedad diacrónica y 2 , el $8,7 \%$, la mantienen de forma parcial. Asimismo, como hemos podido ver en los ejemplos del análisis, la traducción de las expresiones que hemos analizado se ve limitada por el código de colocación de sonido en el doblaje, tanto por la isocronía como por la sincronía.

De los 10 ejemplos que no mantienen o mantienen parcialmente la variedad diacrónica, hay 5 cuya traducción está subordinada a la imagen, es decir, se podría haber usado otra expresión que se acercara más a la variación diacrónica, pero la imagen no lo permite.

A la dificultad de respetar las sincronías e isocronías se une la dificultad de documentación. En primer lugar, es necesario que como traductores seamos capaces de identificar los términos o expresiones característicos de un determinado periodo o época. Si a esto le sumamos 
que en el caso que nos ocupa, este lenguaje era de índole oral, coloquial y, en ocasiones, vulgar, la tarea se vuelve todavía más ardua. Las principales fuentes formales, en este caso los corpus, no registran con tanta frecuencia este tipo de lenguaje, debido, tanto a su volatilidad, como al tiempo que supone su recopilación. Esto hace que en muchas ocasiones tengamos que recurrir a fuentes menos convencionales, cuya fiabilidad no siempre está asegurada.

Una vez identificada la variedad diacrónica llega la segunda dificultad: buscar el modo de respetarla. En este sentido, los corpus han resultado ser una herramienta de gran utilidad, pues nos permiten comprobar cuáles de los equivalentes posibles encajarían en la época objeto de estudio. Como hemos visto, habrá casos en los que no siempre contaremos con un equivalente exacto que logre trasladar al receptor meta al periodo en el que se enmarca la historia. Cuando esto ocurra en numerosas ocasiones, los profesionales de la traducción podrán recurrir a la técnica de la compensación e introducir una de esas expresiones en cualquier otro momento.

No obstante, cabe destacar que en casi ningún caso nos encontraremos un producto en el que sea necesario poner constantemente de manifiesto la variedad diacrónica, sino que, por lo general, los autores suelen simplemente dotar a su obra de ciertos toques que permitan a la audiencia meta identificar el periodo de tiempo en el que se enmarca la obra, pero sin abusar, ya que no se quiere distraer la atención del argumento. Esto último será de gran importancia, ya que el traductor audiovisual deberá tener en cuenta en todo momento el nuevo público al que se dirige y cuál es su conocimiento de la cultura original donde se desarrolla la obra. Los espectadores tendrán, por tanto, un papel determinante en todo el proceso.

\section{REFERENCIAS BIBLIOGRÁFICAS}

Agost, R. (1999). Traducción y doblaje: palabras, voces e imágenes. Barcelona: Ariel Ávila.

Arampatzis, C. (2011). La traducción de la variación lingüística en textos audiovisuales de ficción humorística: dialectos y acentos en la comedia de situación estadounidense doblada al castellano (Tesis doctoral). Universidad de Las Palmas de Gran Canaria, Las Palmas de Gran Canaria. 
Bolaños, S. (2015). La lingüística de corpus: Perspectivas para la investigación lingüística contemporánea. Forma y Función, 28, 3154.

Bolaños, A. (2017). La variación lingüística en subtitulación: el caso de las restricciones en los Amores imaginarios de Xavier Dolan, Entreculturas, 9, 221-237.

Botella, C. (2017). La traducción del humor intertextual audiovisual. Que la fuerza os acompañe, MonTI, 9, 77-100.

Catford, J.C. (1965). A LinguisticTheory of Translation.Londres: Oxford University Press.

Chaume, F. (2004). Cine y traducción. Madrid: Cátedra.

- (2018). Anoverview of audiovisual translation:Four methodological turns in a mature discipline. Journal of Audiovisual Translation, 1(1), 4063.

De los Reyes Lozano, J. (2015). Genética del doblaje cinematográfico. La versión del traductor como proto-texto en el filme Rio. LANS, 14, 149-167

Díaz Cintas, J. (2001). La traducción audiovisual: El subtitulado. Quaderns: Revista de traducció, 9, 169-172.

Fuentes, A. (2000). La recepción del humor audiovisual traducido: estudio comparativo de fragmentos de las versiones doblada y subtitulada al español de la película DuckSoup, de los Hermanos Marx (Tesis doctoral). Universidad de Granada, Granada.

Fuentes, A. (2001). Aspectos profesionales y técnicos de la traducción audiovisual, con especial referencia al caso de España. TRANS: revista de traductología, 5, 143-154.

García, B. y Bolañoz, A. (2018). El análisis de la variación lingüística en el doblaje de la serie Jane theVirgin. Sendebar, 29, 81-107.

Hatim, B. y Mason, I. (1995). Teoría de la traducción. Una aproximación al discurso. Barcelona: Ariel.

Hurtado, A. (2001). Traducción y Traductología. Introducción a la Traducción. ( $6^{a}$ ed.).Madrid: Cátedra.

Kelly, J. (2016, agosto 23). How '80s is the slang in Stranger Things? [Entrada de blog]. Recuperado de http://www.slate.com/blogs/lexicon_valley/2016/08/23/mental_douch 
ebags_chill_wastoids_how_80s_is_the_slang_in_stranger_things.ht 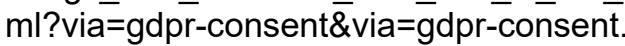

Lazalde, A. (2010, diciembre 17). Hipertextual [Entrada de blog]. Recuperado el 6 de junio de 2018 de https://hipertextual.com/2010/12/google-books-ngram-viewerinvestigacion.

Lomeña, M. (2009). Variación lingüística y traducción para el doblaje: Mujeres al borde de un ataque de nervios. Entreculturas, 1, 275283.

Martínez Sierra, J.J. (2001). El papel del elemento visual en la traducción del humor en textos audiovisuales: ¿Un problema o una ayuda? TRANS: revista de traductología, 13, 139-148.

Marzà, A., Torralba, G., \& Grupo TRAMA (2013). Las normas profesionales de la

traducción para el doblaje en España. Trans, 17, 35-50.

Mayoral, R. (1998). Traducción audiovisual, traducción subordinada, traducción intercultural. En: Seminario de Traducción Subordinada. Sevilla: Facultad de Filología de la Universidad de Sevilla [en línea]. Recuperado el 9 de junio de 2018 de http://www.ugr.es/ rasensio/docs/TAV_Sevilla.pdf.

Mayoral, R. (1999). La traducción de la variación lingüística. Soria: Diputación Provincial de Soria.

Navarro, M. L. (2017). La traducción del humor audiovisual. El caso de la película de animación El espantatiburones (Shark tale). MonTI, 9, 307-329.

Muñoz, R. (1995). Lingüística para traducir. Barcelona: Teide.

Oxford Dictionaries (2017, diciembre, 4). A totally tubular guide to the language of Stranger Things. [Entrada de blog]. Recuperado el 13 de mayo de 2018 de https://blog.oxforddictionaries.com/2017/12/04/stranger-thingslanguage-guide/.

Parrot Analytics (2018). The Global Television Demand Report: Audience title discovery, SVOD platform growth, digital original series popularity and global genre TV demand trends in 2017. Recuperado el 18 de mayo de 2018 en https://insights.parrotanalytics.com/hubfs/Resources/whitepapers/Pa 
rrot $\% 20$ Analytics $\% 20$ -

\%20The\%20Global\%20TV\%20Demand\%20Report\%202017.pdf.

Quin, R. (2017, octubre 27). The totally rad guide to Stranger Things slang. [Entrada de blog]. Recuperado el 27 de abril de 2018 de https://www.collinsdictionary.com/word-lovers-blog/new/the-totallyrad-guide-to-stranger-things-slang, 397,HCB.html.

Rabadán, R. (1991). Equivalencia y traducción: problemática de la equivalencia translémica inglés-español. León: Universidad de León.

Rodríguez, F., et al. (1989). Comunicación y lenguaje juvenil. Madrid: Instituto de Estudios Juan Gil Albert-Editorial Fundamentos.

Rodríguez González, F. (2006). Medios de comunicación y contracultura juvenil. Círculo lingüística aplicada a la comunicación, 25, 5-30.

Tello, I. (2012). Traducción de la variación lingüística: una visión diacrónica. Hikma, 11, 133-159. https://doi.org/10.21071/hikma.v11i.5249.

Uclés, G. (2016). La traducción de las referencias a la variedad diatópica en el doblaje al español. Normas, 6, 77-92.

Vulpoi, E. L. (2018). La traducción audiovisual y el perfil del traductor audiovisual en Rumanía (Tesis doctoral). Universitat Jaume I, Castelló de la Plana. Recuperado de https://www.tdx.cat/bitstream/handle/10803/461062/2018_Tesis_\%2 OVulpoiu_Elena.pdf?sequence $=1$

Zamora, P. (2018). El doblaje y la traducción literaria de El Capitán Alatriste en italiano. Sendebar, 29, 59-79.

\section{DICCIONARIOS Y CORPUS UTILIZADOS}

American HeritageDictionary. Recuperado el 9 de junio de 2018 de https://www.ahdictionary.com/.

Costea, E. (n.d). Corpus of Historical American English [Entrada de blog]. Recuperado el 6 de junio de 2018 de http://www.helsinki.fi/varieng/CoRD/corpora/COHA/.

Davies, M. Corpus of Contemporary American English (COCA) [en línea]. Recuperado el 9 de junio de 2018 de https://corpus.byu.edu/coha/.

Davies, M. Corpus of Historical American English (COHA) [en línea]. Recuperado el 9 de junio de 2018 de https://corpus.byu.edu/coha/. 
Google Books Ngram Viewer [en línea]. Recuperado el 9 de junio de 2018 de https://books.google.com/ngrams.

Merriam Webster Dictionary. Recuperado el 9 de junio de 2018 de https://www.merriam-webster.com/.

Oxford English Dictionaries. Recuperado el 9 de junio de 2018 de https://en.oxforddictionaries.com/.

Real Academia Española (2001). Diccionario de la Lengua Española. Recuperado el 9 de junio de 2018 de http://dle.rae.es/.

Real Academia Española: Banco de datos (CORDE) [en línea]. Corpus diacrónico del español. Recuperado el 8 de junio de 2018 de http://www.rae.es.

Real Academia Española: Banco de datos (CREA) [en línea]. Corpus de referencia del español actual. Recuperado el 8 de junio de 2018 de http://www.rae.es.

Real Academia Española (2018). Recursos. Recuperado el 6 de junio de 2018 de http://www.rae.es/recursos/banco-de-datos.

The Free Dictionary. Recuperado el 9 de junio de 2018 de https://es.thefreedictionary.com/.

Urban Dictionary. Recuperado el 9 de junio de 2018 de https://www.urbandictionary.com/.

Wordnik. Recuperado el 9 de junio de 2018 de https://www.wordnik.com/.

YourDictionary. Recuperado el 9 de junio de 2018 de http://www.yourdictionary.com/. 